\title{
Caracterización morfológica de aislados de Colletotrichum asociados a antracnosis en cítricos en Tuxpan Veracruz
}

Morphological characterization of Colletotrichum isolates associated to citrus anthracnose in Tuxpan, Veracruz

Allende-Molar Raúl ${ }^{1 凶}$, Alarcón-Pulido Sara Aída ${ }^{1}$, Hernández-Sánchez María de la Luz¹, GonzálezCárdenas Julio César ${ }^{1}$, Elorza-Martínez Pablo ${ }^{1}$

Facultad de Ciencias Biológicas y Agropecuarias. Universidad Veracruzana. Zona Poza Rica- Tuxpan

${ }^{\square}$ Autor para correspondencia: raallende@uv.mx

Recibido: $17 / 10 / 2019$

Aceptado: $12 / 11 / 2019$

\section{RESUMEN}

El objetivo de este trabajo fue aislar y caracterizar morfológicamente aislados de Colletotrichum responsables de antracnosis en cítricos en Tuxpan Ver. Se utilizaron muestras de tejidos vegetales con síntomas de antracnosis para obtener aislados de Colletotrichum, una vez obtenidos se codificaron y se hicieron bioensayos arreglados en un diseño completamente al azar con 6 tratamientos y 4 repeticiones para observar el desarrollo de cada aislado. Cada uno de los aislados obtenidos fue considerado como un tratamiento Las variables analizadas fueron tasa de crecimiento, caracterización de colonias y de apresorios. Los resultados mostraron que existieron diferencias significativas entre los tratamientos: en cuanto a su crecimiento el aislado UVA1 fue el que mostró la menor tasa de crecimiento de $6.5 \mathrm{~mm}$ por día, su desarrollo de micelio fue blanco casi transparente sin presencia de masas conidiales y en los otros aislados el crecimientos casi siempre fue similar con rangos de la tasa de crecimiento por día entre 8.1 a $9.0 \mathrm{~mm}$ por día; en cuanto a la caracterización de colonias fueron casi similares ya que el micelio en la myoría fue abundante con masa conidiales de color salmón y masas de esclerocios de color negro. La formación de apresorios ocurrió entre 24 y 72 h.

Palabras clave: apresorios, masas conidiales, enfermedades, fitopatógeno

\begin{abstract}
The objective of this work was to isolate and characterize morphologically Colletotrichum isolates responsible of citrus anthracnose in Tuxpan, Veracruz. Plant tissues with anthracnose symptoms were used to obtain Colletotrichum isolates, after that a code was assigned to each isolate. A bioassay arranged in a completely randomized design included 6 treatments and 4 repetitions was conducted to observe the development of each isolate. The analyzed variables were growth rate, colony and appressoria characteristics. Results showed that there were statistically significant differences between treatments: the isolate UVA1 showed the lowest growth rate $(6.5 \mathrm{~mm} /$ day $)$, with white mycelia and without spore masses; in the remaining isolates the growth rate was similar with values between 8.1 and $9.0 \mathrm{~mm} / \mathrm{day}$; the colony characteristics were similar with abundant mycelia and black sclerotial masses. Appressoria were formed between 24 and $72 \mathrm{~h}$.
\end{abstract}


Keywords: appressoria, conidial masses, diseases, plant pathogen.

\section{INTRODUCCIÓN}

Las enfermedades causadas por hongos patógenos, representan uno de los factores limitantes para la producción de los cultivos (Trigriano et al., 2004). En el caso de los cítricos, el hongo Colletotrichum causa la enfermedad conocida como antracnosis; la enfermedad causa entre otros daños: caída de frutos, muerte regresiva de ramas y lesiones en hojas. La reducción en la producción causada por esta enfermedad se ha documentado que es entre el 15 y $30 \%$ y en situaciones climáticas de alta humedad relativa y temperaturas altas la incidencia y daños pueden ser mayores (Guarnaccia et al., 2017). Esta enfermedad se encuentra distribuida en todas las regiones productoras de México con ataques severos en floración, fructificación y poscosecha (Becerra, 1995).

Actualmente a nivel mundial, se han reportado alrededor de 23 especies de Colletotrichum patogénicas en cultivos de cítricos (Ramos et al., 2016; Guarnaccia et al., 2017). En china, se identificaron nueve especies de Colletotrichum como causantes de antracnosis en cítricos: $C$. gloeosporioides, C. fructicola, C. karstii, C. citricola, C. citri, C. brevispora, C. boninense, C. simmonsdi, and C. murrayae (Peng et al., 2012; Huang et al., 2013). En el estudio más reciente en Europa, tres nuevas especies fueron identificadas $C$. novae-zelandiae, $C$. catinaense and C. limonicola (Guarnaccia et al., 2017). Estos cambios recientes, así como la detección e identificación de nuevas especies patogénicas en cítricos, justifican la realización de estudios que impliquen nuevos muestreos y la caracterizacion de aislados que causen antracnosis en regiones poco estudiadas. Estos estudios podrian ayudar a predecir los riesgos de resistencia a fungicidas y a explicar las diferencias en patogenicidad y características morfológicas entre especies de Colletotrichum (Orozco, 2006).

La zona norte de Veracruz representa un área importante en la producción de cítricos; sin embargo, no existen estudios comparativos en morfología, patogenicidad y genética de poblaciones del hongo reposnable de antracnosis. Por ello el objetivo de este trabajo fue caracterizar aislados de Colletotrichum asociados a antracnosis en cultivos de cítricos establecidos en la zona de Tuxpan, en el norte del estado de Veracruz.

\section{MATERIALES Y MÉTODOS}

Colecta de muestras. La recolección de muestras de antracnosis se llevó a cabo en distintas localidades del municipio de Tuxpan. Se colectaron hojas, ramas y frutos con síntomas típicos de antracnosis en huertos de cítricos. Cada muestra (10 en total) se colocó en una bolsa de papel, se depositó en una hielera y se transportó al laboratorio.

\section{Aislamiento, purificación y preservación de aislados de Colletotrichum. De los frutos, hojas y ramas con síntomas de antracnosis, se seleccionaron porciones de tejido de aproximadamente $0.5 \mathrm{~cm}$ del área de avance de la lesión, se desinfestaron en una solución de cloro al $3 \%$ durante 2 min, después se colocaron en alcohol etílico durante $30 \mathrm{~s}$; posteriormente se}


colocaron en agua destilada por 1 minuto y finalmente, se colocaron en papel absorbente. Las muestras desinfestadas se colocaron en placas de medio de cultivo papa dextrosa agar (PDA) suplementado con cloranfenicol (0.25 $\mathrm{g} / \mathrm{l}$ ) y se incubaron a $25^{\circ} \mathrm{C}$ (Aiello et al., 2015). La purificación se llevó a cabo mediante la técnica de transferencia de puntas de hifas la cual consistió en transferir con la ayuda de una aguja esterilizada una nueva porción de micelio de la colonia en crecimiento activo a nuevas cajas Petri con. Una vez que se obtuvieron los aislados purificados, la preservacion se realizó al colocar un disco de crecimiento micelial del aislado purificado, de colonias de al menos 7 días de edad, en tubos de ensayo con medio de cultivo PDA inclinado.

Caracterización morfologica de aislados. La caracterización morfológica de los aislados de Colletotrichum incluyo el registro de crecmiento micelial en medio de cultivo y el aspecto de la colonia (Orozco, 2006). La tasa de crecimiento de todos los aislados se determinó de la siguiente manera: Discos miceliales de $8 \mathrm{~mm}$ de diámetro se tomaron de áreas en crecimientoactivo de colonias de 6 días de edad y se transfirieron a placas Petri de $8 \mathrm{~cm}$ de diámetro con agar papa dextrosa (PDA), las cuales se incubaron a $25^{\circ} \mathrm{C}$ se evaluaron cuatro réplicas de cada aislado. $\mathrm{El}$ diámetro de la colonia se midió diariamente durante 8 días y se calculó la tasa de crecimiento en $\mathrm{mm}$ por día. Adicionalmente se registró el color de la colonia, la textura y la presencia de masas conidiales (Ramos et al., 2016).
Inducción de formación de apresorios. Los apresorios conidiales se indujeron al colocar conidios en dos gotas de agua destilada sobre un portaobjetos de vidrio. Los portaobjetos se colocaron dentro de un placa Petri que contenía algodón humedecido con agua destilada esterilizada y se incubaron a $25^{\circ} \mathrm{C}$ en oscuridad (Peng et al., 2012).

Diseño experimental y análisis estadístico. La tasa de crecimiento de los aislados obtenidos se analizó bajo un diseño estadístico completamente al azar. Cada aislado se consideró como un tratamiento y cada tratamiento tuvo 4 repeticiones. La variable experimental fue el diámetro de la colonia, la cual se midió diariamente durante 8 días y se calculó la tasa de crecimiento en mm por día.

\section{RESULTADOS Y DISCUSIÓN}

En los recorridos por las parcelas citrícolas de Tuxpan, Veracruz se observaron los síntomas típicos de antracnosis: Las hojas presentaron manchas necróticas a diferencia del fruto la expresión de Colletotrichum se manifiesta con puntos concéntricos de color café oscuro, las manchas crecían paulatinamente hasta unirse y necrosar (Figura 1). Esto concuerda con Orozco et al. (2006) quienes mencionan que los síntomas presentados en hojas y frutos son similares a los de nuestras muestras. Se colectaron un total de 10 muestras con síntomas de antracnosis de las cuales se logró el aislamiento de 6 (Cuadro 1).

Cuadro 1. Aislados de Colletotrichum obtenidos de infecciones de antracnosis en tejidos de cítricos de Tuxpan Veracruz

\begin{tabular}{llll}
\hline Código & Tejido & Huésped & Localidad \\
\hline UVA1 & Hoja & Toronja & Higueral \\
\hline
\end{tabular}




\begin{tabular}{llll}
\hline UVA3 & Hoja & Naranja & Coloman \\
UVA5 & Hoja & Naranja & La camelia \\
UVA6 & Fruto & Toronja & Higueral \\
UVA7 & Hoja & Limón & Cañada Rica \\
UVA10 & Fruto & Mandarina & Buenos Aires \\
\hline
\end{tabular}

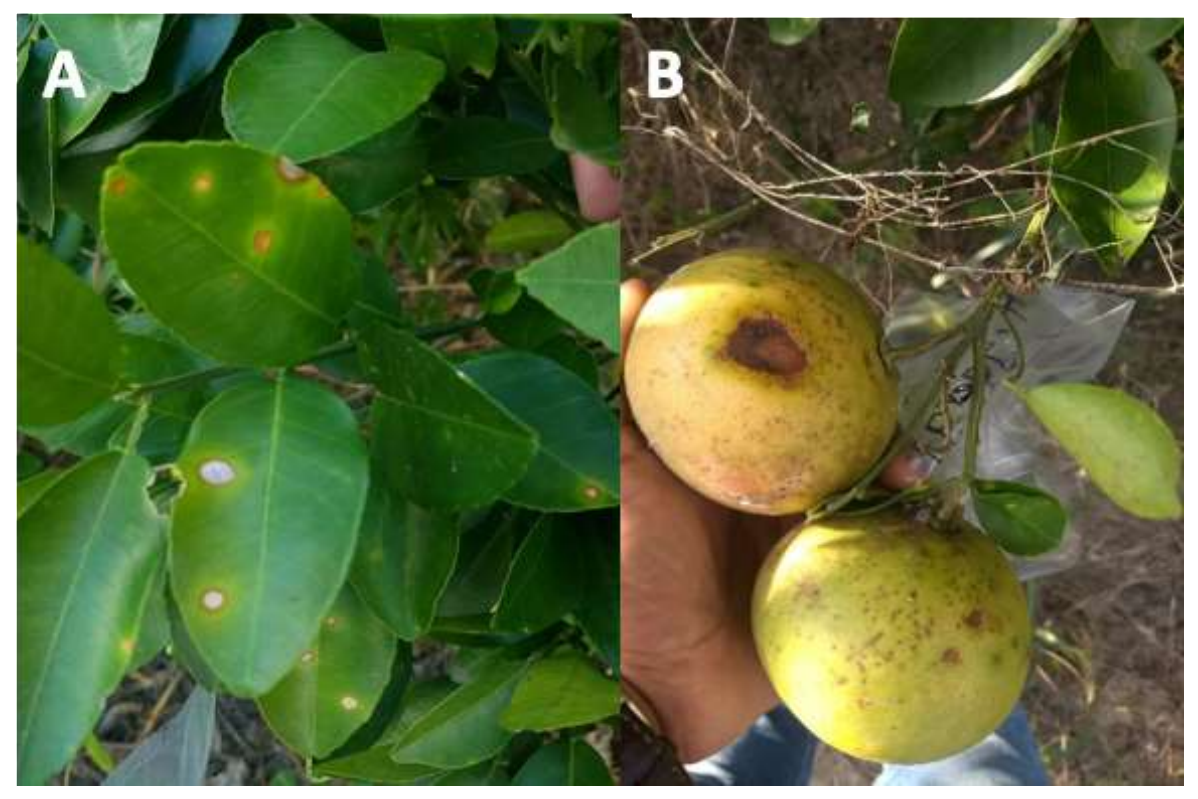

Figura 1. Síntomas de antracnosis. A: En hojas de C. sinensis B. En fruto de C. paradisi

Las colonias de los hongos presentaron diferencias morfológicas durante su observación durante 8 días de edad, ya que existió variación en su tamaño de crecimiento en el medio de cultivo PDA. Los aislados de Colletotrichum a los 8 días de crecimiento mostraron diferencias estadísticas significativas ya que los aislados UVA5, UVA7 y UVA10 tuvieron el mayor desarrollo y el aislado UVA1 fue el que presentó menor crecimiento (Figura 2). 


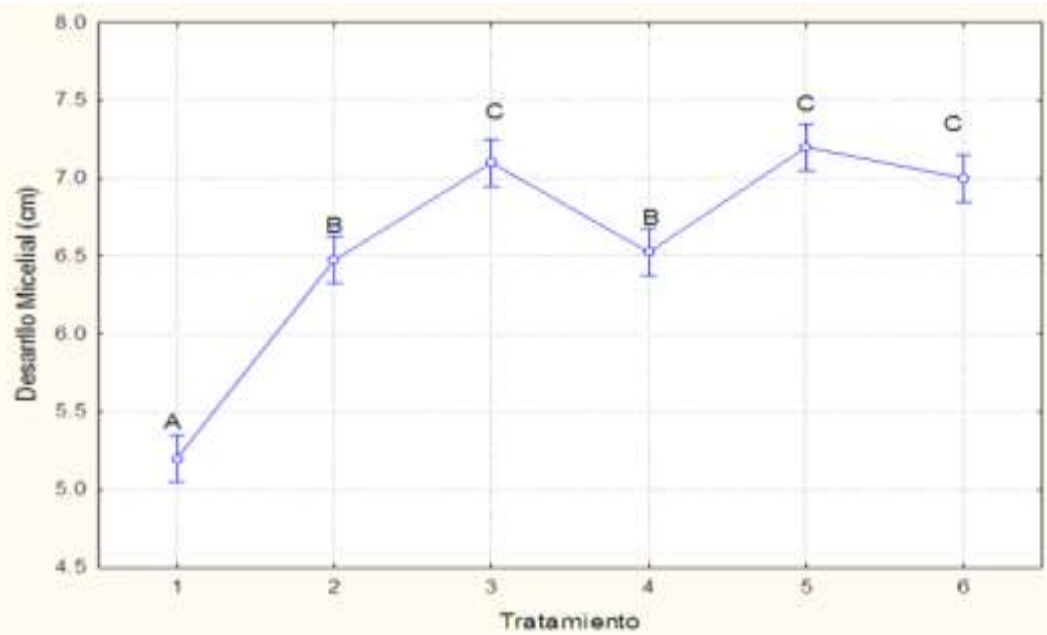

Figura 2. Crecimiento de aislados de Colletotrichum en medio de cultivo PDA a los 8 días. Las medias con letras distintas son estadísticamente diferentes de acuerdo a la prueba de Tukey $(\mathrm{P}=0.05)$. Tratamientos (aislados) 1=UVA1, 2=UVA3, 3=UVA5, 4=UVA6, 5=UVA7, 6=UVA10.

El aislado UVA7 fue el que tuvo mayor tasa de crecimiento con una tasa de $9.0 \mathrm{~mm}$ por día; mientras que, el UVA1 tuvo un crecimiento de $6.5 \mathrm{~mm}$ por día (Cuadro 2). Las tasas de crecimiento de los aislados de Colletotrichum provenientes de cítricos fue menor a la reportada por Santacruz (2013) cuando evaluó el crecimiento en aislados de Colletotrichum en ají y encontró tasas que fluctuaron entre 7 y 13 $\mathrm{mm} /$ día.

Cuadro 2. Tasa de crecimiento micelial de los aislados de Colletotrichum provenientes de cítricos de Tuxpan, Veracruz.

\begin{tabular}{cc}
\hline Aislados & $\mathrm{mm} /$ día \\
\hline UVA1 & 6.5 \\
UVA3 & 8.1 \\
UVA5 & 8.9 \\
UVA6 & 8.2 \\
UVA7 & 9.0 \\
UVA10 & 8.8 \\
\hline
\end{tabular}

Caracterización de colonias. En la mayoría se observaron halos concéntricos con presencia de masas de esporas, con excepcion del islado UVA1 y UVA6 en los que no se observaron masas de esporas. Se manifestaron diferentes tipos de texturas en las colonias, tales como algodonosas, poco densas, con masas de esclerocios color negro y masa de esporas color naranja- salmón (Figura 3). Estos resultados son similares a los observados por Orozco (2006) en antracnosis en cítricos. Las masas conidiales de color salmón y negras en distribución concéntrica con micelio abundante y algodonoso de color blanco fueron reportadas tambien por 
Trinidad et al. (2017) y Santacruz (2013) y nuestro estudio. observadas en los aislados UVA3 y UVA10 de

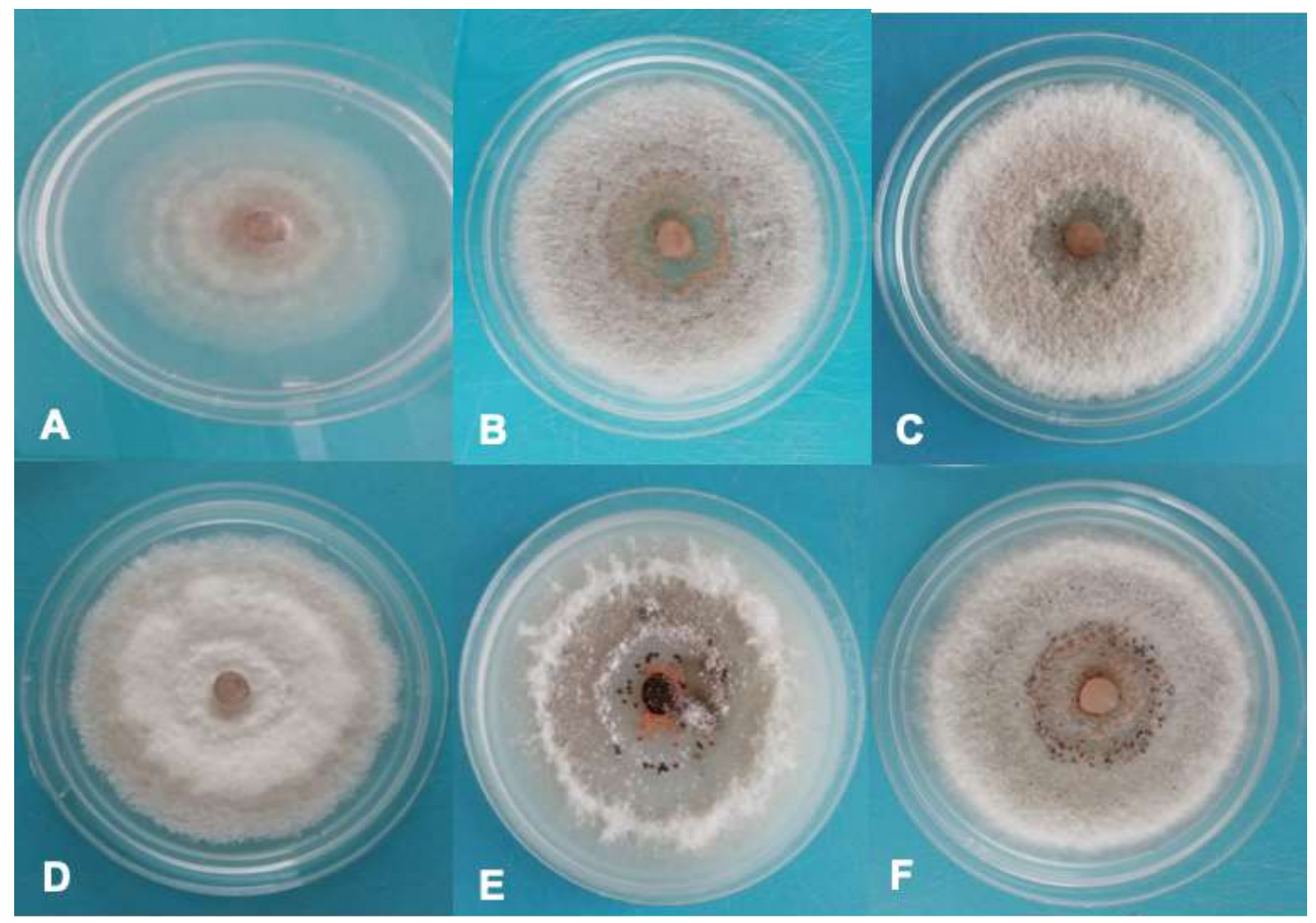

Figura 3. Aislados de Colletotrichum a los 8 días de crecimiento en medio de cultivo PDA. A= UVA1, $\mathrm{B}=\mathrm{UVA} 3, \mathrm{C}=\mathrm{UVA}$ 5, $\mathrm{D}=\mathrm{UVA} 6, \mathrm{E}=\mathrm{UVA} 7, \mathrm{~F}=\mathrm{UVA} 10$

Caracterización de apresorios. A las 24 horas los aislados UVA1, UVA5, UVA6 y UVA7 mostraron la presencia de apresorios; a las 48 se observaron en el aislado UVA3 y en el aislado UVA10 los apresorios se observaron a las $72 \mathrm{~h}$ (Figura 4). Los apresorios observados tuvieron una forma lobulada y globosa; esta forma concuerda con Orozco (2006) quien obtuvo apresorios de forma lobulados en aislamientos de C. gloeosporioides causantes de antracnosis en limón. La formación temprana $(24$ h) de apresorios en los aislados UVA1, UVA5, UVA6 y UVA7 podría significar una mayor virulencia o capacidad de infección pues los apresorios están relacionados con la penetración directa que hace el hongo Colletotrichum en los tejidos susceptibles de infección. 


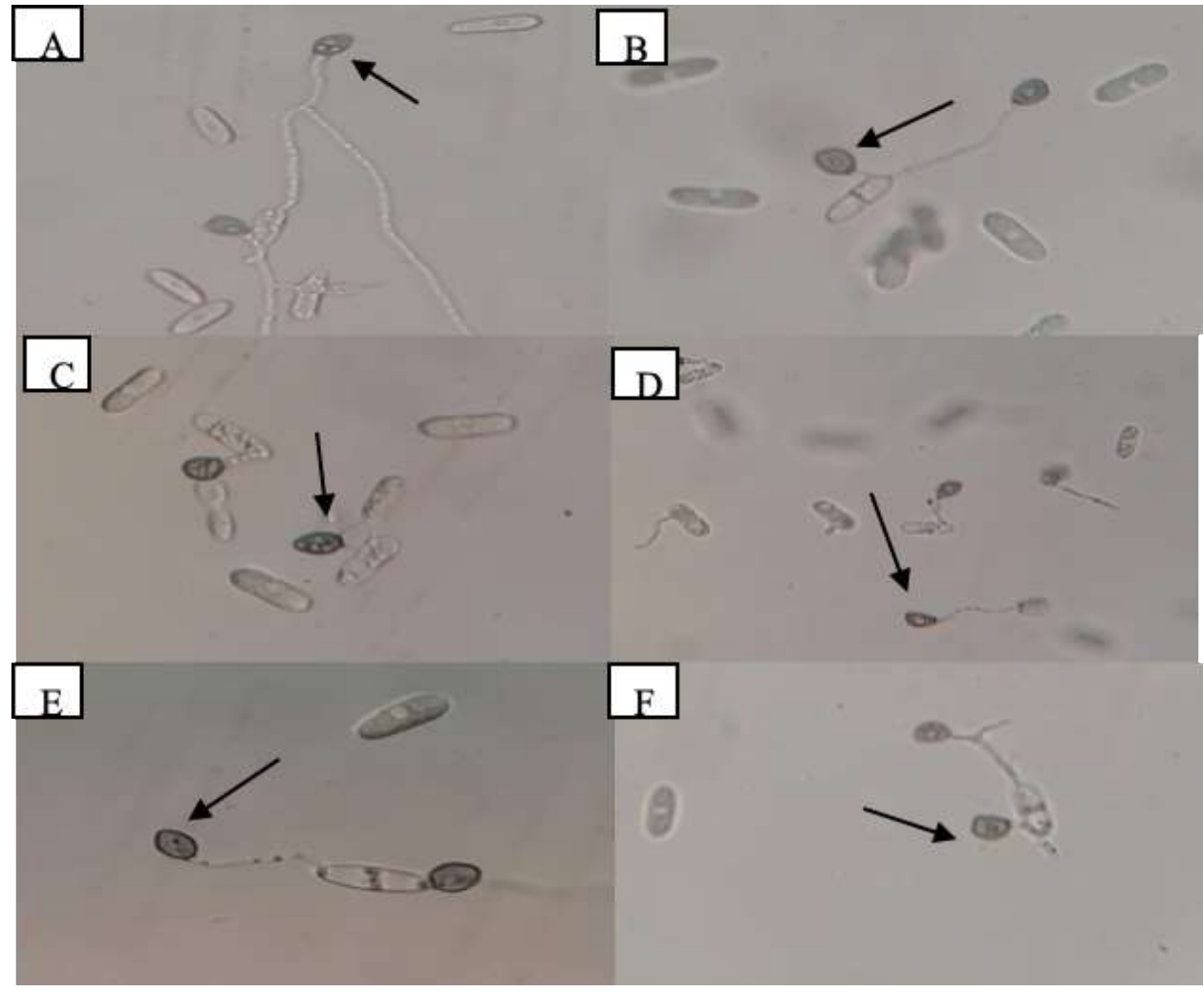

Figura 4. Apresorios de aislados de Colletotrichum provenientes de antracnosis en cítricos en Tuxpan, Veracruz. A=UVA1, B=UVA3, C=UVA5, D=UVA6, E=UVA7, F=UVA10

\section{CONCLUSIONES}

En este estudio se evidenció que existe variabilidad morfológica en los aislados de Colletotrichum asociados a infecciones de antracnosis en cítricos en Tuxpan, Veracruz. Se obtuvieron más aislados a partir de hojas ya que en este tejido las lesiones son más visibles y frecuentes. De acuerdo al trabajo realizado, se observó que existe variabilidad: en la morfología de colonias, ya que existieron colonias con micelio aéreo y otras con micelio ralo; en el crecimiento, ya que la tasa de crecimiento varió entre 6.5 y $9.0 \mathrm{~mm} /$ día; y en la formación de apresorios ya que su producción varió desde 24 a $72 \mathrm{~h}$. Este primer estudio de la variabilidad morfológica de aislados de Colletotrichum asociados a antracnosis de cítricos en Tuxpan,
Veracruz servirá de base para estudios futuros relacionados con la identificación de especies, su distribución en la zona y su sensibilidad a los fungicidas.

\section{AGRADECIMIENTOS}

A la SEP/DGESU por el financiamiento al proyecto PRODEP UV-PTC-872.

\section{LITERATURA CITADA}

Aiello D, Carrieri R, Guarnaccia V, Vitale A, Lahoz E and Polizzi G. 2015. Characterization and pathogenicity of Colletotrichum gloeosporioides and $C$. karstii causing preharvest disease on 
Citrus sinensis in Italy. Journal of Phytopathology 163:168-177

https://doi.org/10.1111/jph.12299

Becerra, L. E. N. 1995. Enfermedades del cultivo de mango. pp. 83-101. En: Inocente Mata Beltrán y Raúl Mosqueda Vázquez (eds.). La Producción de Mango en México. Noriega Editores. México, D.F. 159 p.

Guarnaccia V, Groenewald JZ, Polizzi G, Crous PW. 2017. High species diversity in Colletotrichum associated with citrus diseases in Europe. Persoonia 39:32-50 https://doi.org/10.3767/persoonia.2017.39. 02

Huang F, Chen GQ, Hou X, Fu YS, Cai L, Hyde KD, Li HY. 2013. Colletotrichum spp. associated with cultivated Citrus in China. Fungal diversity 61:61-74 https://doi.org/10.1007/s13225-013-0232

Orozco S. M. (2006). Patogenicidad, variabilidad morfológica y genética de Colletotrichum acutatum Simmonds de cítricos en México. Tesis de Doctorado en Ciencias. Universidad de Colima 91 pp.

Orozco S. M., Medina U.V.M., Robles G. M., Orozco R. J., Pérez Z. O., Velázquez M.J.J., Timmer, L.W. y Guzmán G. S. (2006). Biología y manejo integrado de antracnosis del limón mexicano en el trópico seco de México. SAGARPA, INIFAP, CIRPAC. Campo Experimental Tecomán. Folleto Técnico Núm. 2. 73 p.

Peng LJ, Yang Y, Hyde KD, Bahkali AH, and Liu Z. 2012. Colletotrichum species on Citrus leaves in Guizhou and Yunnan provinces, China. Cryptogamie, Mycologie 33:267-283

https://doi.org/10.7872/crym.v33.iss3.201

2.267
Ramos AP, Thalinhas P, Sreenivasaprasad S, Oliveira H. 2016. Characterization of Colletotrichum gloeosporioides, as the main causal agent of citrus anthracnose, and $C$. karstii as species preferentially associated with lemon twig dieback in Portugal. Phytoparasitica 44:549-561 https://doi.org/10.1007/s12600-016-0537$\mathrm{y}$

Santacruz D. C. X 2013. Caracterización morfológica, patogénica y molecular de especies de Colletotrichum spp. causantes de la antracnosis del fruto de ají y pimentón capsicum spp. en el valle del cauca. Tesis de Maestría en Ciencias Agrarias. Universidad Nacional de Colombia. 97pp.

Trigriano, R.N., Windham, M.T., and Windham, A.S. (Eds.). 2004. Plant Pathology. Concepts, and laboratory excercises. CRC Press. Boca Raton, Florida, USA. 413 p.

Trinidad A.E., Ascencio V.F., Ulloa J.A., Ramírez R.J.C., Ragazzo S.J.A., Calderón S.M. y Bautista R.P.U. (2017). Identificación y caracterización de Colletotrichum spp. causante de antracnosis en aguacate Nayarit, México. Revista Mexicana de Ciencias Agrícolas, 19 , 12. https://doi.org/10.29312/remexca.v0i19.66 4 
Copyright (c) 2019 Raúl Allende-Molar, Sara Aida Alarcón-Pulido, Maria de la Luz Hernández-Sánchez, Julio César González-Cár denas, Pablo Elorza Martinez

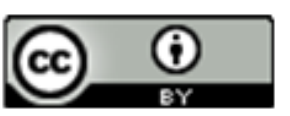

Este texto está protegido por una licencia licencia CreativeCommons 4.0.

Usted es libre para Compartir — copiar y redistribuir el material en cualquier medio o formato-y Adaptar el documento — remezclar, transformar y crear a partir del material- para cualquier propósito, incluso para fines com erciales, siempre que cumpla la condición de:

Atribución: Usted debe dar crédito a la obra original de manera adecuada, proporcionar un enlace a la licencia, e in dicar si se han realizado cambios. Puede hacerlo en cualquier forma razonable, pero no de forma tal que sugiera que tiene el apoyo del licenciante o lo recibe por el uso que hace de la obra.

Resumendelicencia - Textocompletodelalicencia 